oxygenation, which may play a role in the multifactorial pathophysiology of necrotizing enterocolitis (NEC).

The aim of this study is to evaluate by NIRS the effects of bolus and continuous enteral feeding on splanchnic and cerebral oxygenation in preterm infants with normal feeding tolerance.

Methods Eighteen healthy preterms (GA 27-32 weeks), tolerating at least $100 \mathrm{ml} / \mathrm{kg}^{-1} / \mathrm{day}^{-1}$ of fortified human milk or preterm formula, underwent a 6-hours simultaneous monitoring of cerebral and splanchnic oxygenation using NIRO-200 oximeter. Sensors were placed on frontal and sub-umbilical region. During the monitoring they randomly received a 10 -minutes bolus meal and a 3 hours continuous meal.

Recorded values of cerebral and splanchnic Tissue Oxygenation Index (TOI) were clustered in 5-minutes intervals and compared between different feeding techniques using Wilcoxon Signed Ranks Test. Statistical significance was set at $p \leq 0.05$.

Results Splanchnic oxygenation significantly decreased $(p<0.05)$ during continuous feeding, from 1.30' hour after the beginning to almost the end of the feed. No differences were found on cerebral oxygenation.

Conclusions To the best of our knowledge, this is the first study comparing the effect of different feeding techniques on splanchnic and cerebral oxygenation in preterms. A significant reduction in splanchnic oxygenation was observed during continuous enteral. A possible role of these findings on the multifactorial NEC pathogenesis remains to be investigated. \\ IS FOLIC ACID SUPPLEMENTATION REALLY NECESSARY IN PRETERM INFANTS WITH $\leq 32$ WEEKS OF GESTATION?}

doi:10.1136/archdischild-2012-302724.0347

'MY Oncel, 'E Calisici, 'R Ozdemir, 'S Yurttutan, 10 Erdeve, ${ }^{2} \mathrm{~S}$ Karahan, 1,3U Dilmen. ${ }^{1}$ Neonatology, Zekai Tahir Burak Maternity Teaching Hospital; ${ }^{2 B}$ Biostatistics, Hacettepe University, Faculty of Medicine; ${ }^{3}$ Pediatrics, YIldırm Beyazıt University, Faculty of Medicine, Ankara, Turkey

Background and Aims The main goal of this study was to determine the necessity of folic acid supplementation in preterm infants. Methods Infants born $\leq 32$ weeks of gestation were included in the study with parental consent. Blood samples for the determination of serum folate levels were obtained on days 14 and 28 postnatally, as well as 36 weeks postconceptionally (or just before discharge if patients are discharged before 36 weeks) - samples A, B and C, respectively. Infants were divided into three groups based on mode of feeding; human breast milk (HBM), fortified HBM or preterm formula (PF).

Results A total of 162 preterm infants were enrolled in the study, $17(10.5 \%)$ of whom received HBM alone, $94(58 \%)$ received fortified HBM and 51(31.5\%) were fed with PF. Comparisons between groups revealed that preterm infants in the fortified HBM and PF groups to have significant higher serum folic acid levels in samples $\mathrm{C}$ compared to those receiving HBM alone ( $\mathrm{p}<0.001$ for both). None of the preterm infants included developed folate deficiency during the study period.

Conclusion This is the largest and most comprehensive clinical study to date evaluating the need for folic acid supplementation in preterm infants who were fed using either modern PFs or milk fortifiers mixed with HBM. Our results suggest that fortified HBM use in preterm infants can alleviate the need for further folic acid supplementation. On the other hand, in preterm infants who are unable to receive HBM folic acid support can be provided with PFs.

\section{EFFECTS OF GLUTAMINE SUPPLEMENTATION ON BRAIN DEVELOPMENT OF VERY PRETERM CHILDREN: A FOLLOW- UP STUDY AT SCHOOL-AGE}

doi:10.1136/archdischild-2012-302724.0348
1.JF de Kieviet, ${ }^{2,3}$ RM van Elburg, ${ }^{4}$ PJW Pouwels, ${ }^{1} \mathrm{PJ}$ Vuijk, ${ }^{2} \mathrm{G}$ Boehm, ${ }^{3} \mathrm{~A}$ van Zwol, ${ }^{3} \mathrm{~A}$ van den Berg, ${ }^{3} \mathrm{HN}$ Lafeber, ${ }^{5} \mathrm{RJ}$ Vermeulen, ${ }^{1} \mathrm{~J}$ Oosterlaan. ${ }^{1}$ Clinical Neuropsychology, VU University, Amsterdam; ${ }^{2}$ Centre for Specialized Nutrition, Danone Research, Wageningen; ${ }^{3}$ Paediatrics; ${ }^{4}$ Physics and Medical Technology; ${ }^{5}$ Paediatric Neurology, VU University Medical Center, Amsterdam, The Netherlands

Background and Aims The amino acid glutamine has been shown to reduce the number of serious neonatal infections in very preterm children ( $<32$ weeks of gestation), which may benefit long term brain development. The aim of this study was to elucidate potential effects of glutamine supplementation on brain development in very preterm children at school-age.

Methods First, we investigated growth trajectories of head circumference, weight, and length in the first year for 65 very preterm children that originally participated in a randomized controlled trial on enteral glutamine supplementation between day 3 and 30 of life. Second, we measured brain structure volumes and white matter integrity for 52 very preterm children at school-age, using magnetic resonance imaging (MRI) and Diffusion Tensor Imaging (DTI), respectively. Furthermore, differences in functional outcomes were explored. Group differences were tested using ANOVA statistics.

Results Glutamine supplementation was associated with improved growth trajectories of head circumference in the first year of life $(\mathrm{d}=0.66, \mathrm{p}=0.03)$. Furthermore, glutamine supplementation increased white matter $(\mathrm{d}=0.54, \mathrm{p}=0.03)$, hippocampus $(\mathrm{d}=0.47$, $\mathrm{p}=0.02)$, and brain stem $(\mathrm{d}=0.54, \mathrm{p}=0.04)$ volumes at school-age. All differences were strongly related with the number of serious neonatal infections (all $\mathrm{p}<0.02$ ). Glutamine supplementation did not influende measures of motor, cognitive, and behavioral functioning at school-age.

Conclusions We found evidence that reduction of serious infections by neonatal glutamine supplementation improves head growth in the first year of life, as well as brain structure volumes at school-age. This suggests an early programming effect of nutritional intervention with enteral glutamine.

\section{EFFECTS OF POSTNATAL ENERGY AND MACRONUTRIENT INTAKES ON GROWTH IN EXTREMELY PRETERM INFANTS: A POPULATION-BASED STUDY}

doi:10.1136/archdischild-2012-302724.0349

${ }^{1} E$ Stoltz Sjöström, 'I Öhlund, ${ }^{2} \mathrm{~F}$ Ahlsson, ${ }^{3} \mathrm{M}$ Norman, ${ }^{4}$ E Engström, ${ }^{4} \mathrm{~A}$ Hellström, ${ }^{5} \mathrm{~V}$ Fellman, ${ }^{5,6} \mathrm{E}$ Olhager, ${ }^{1} \mathrm{M}$ Domellöf. ${ }^{1}$ Dept. of Clinical Sciences, Pediatrics, Umeå University, Umeå; 'Dept. of Women's and Children's Health, Uppsala University, Uppsala; ${ }^{3}$ Dept. of Clinical Science, Intervention \& Technology, Karolinska Institute, Stockholm; IInstitute of Clinical Sciences, Section for the Health of Women and Children, Sahlgrenska University, Gothenburg; ${ }^{5}$ Dept. of Pediatrics, Clinical Sciences, Lund University, Lund; 'Dept. of Pediatrics, Linköping University, Linköping, Sweden

Background Extremely preterm infants often experience growth failure and adequate nutritional supply may be difficult to achieve. It is still debated to what extent nutrition affects growth at an early stage in life. The aim of this study was to explore associations between energy and macronutrient intakes and growth.

Methods The study population consists of extremely preterm infants born in Sweden during 2004-2007. Detailed data of nutritional intakes and anthropometric measurements were retrieved from hospital records.

Results Infants ( $\mathrm{n}=602$ ) had a mean \pm SD gestational age of $25.3 \pm 1.1$ weeks and birth weight $765 \pm 171 \mathrm{~g}$. From birth to 70 days of age, energy and protein intakes were $119.3 \pm 11.3 \mathrm{kcal} / \mathrm{kg} /$ day and $3.2 \pm 0.4 \mathrm{~g} / \mathrm{kg} /$ day respectively. Infants showed postnatal growth failure: mean standard deviation scores (SDS) decreased by 1.5 for weight, 2.3 for length and 0.8 for head circumference.

The following confounders were included in the multivariate analyses: Gestational age, CRIB-score, duration of mechanical ventilation, days on postnatal steroid and antibiotics treatment, infant 